\title{
Erratum to: Leaf area lost, rather than herbivore type, determines the induction of extrafloral nectar secretion in a tropical plant (Clerodendrum philippinum)
}

Fang Fang $\mathrm{Xu}^{1} \cdot \mathrm{Jin}^{\mathrm{Chen}}{ }^{1} \cdot$ John Husson ${ }^{2}$

Published online: 30 April 2015

(C) Springer Science+Business Media Dordrecht 2015

Erratum to: Arthropod-Plant Interactions (2014) 8:513-518

DOI 10.1007/s11829-014-9331-2

In the original publication of the article, the $y$ axis of the Fig. 1 should be " $S_{\text {After herbivore }}-S_{\text {Before herbivore", not }}$ " $S_{\text {Before herbivore }}-S_{\text {After herbivore". The corrected Fig. } 1 \text { is }}$ given below.
The online version of the original article can be found under doi:10.1007/s11829-014-9331-2.

\section{Jin Chen}

cj@xtbg.org.cn

1 Key Laboratory of Tropical Forest Ecology, Xishuangbanna Tropical Botanical Garden, Chinese Academy of Sciences, Mengla 666303, Yunnan, China

2 Undergraduate Program, Middlebury College, Middlebury, VT 05753, USA

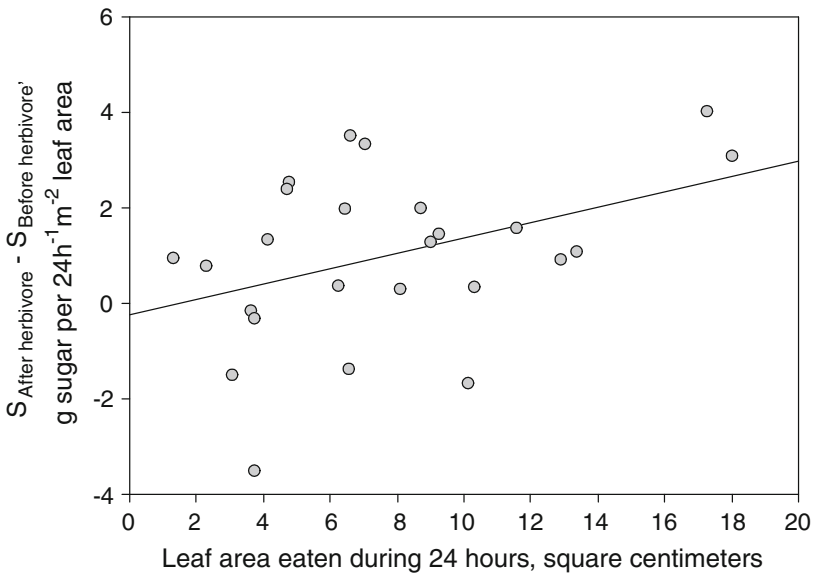

Fig. 1 Regression of the change of EFN secretion rate on the leaf area eaten by herbivores $\left(R_{\text {adj }}^{2}=0.12, F_{1,23}=4.38, P=0.0475\right)$. The change of the EFN secretion was calculated as $S_{\text {After herbivore }}-$ $S_{\text {Before herbivore, }}$ where $S_{\text {After herbivore }}=$ the EFN secretion rate after herbivore treatment, $S_{\text {Before herbivore }}=$ the EFN secretion rate before herbivore treatment, measured 1 day before herbivore treatment 